\title{
Effect of corticosteroid therapy in the early phase of acute respiratory distress syndrome: a propensity- matched cohort study
}

\author{
Moon Seong Baek ${ }^{1}$, Yunkyoung Lee ${ }^{2}$, Sang-Bum Hong ${ }^{2}$, Chae-Man Lim², Younsuck Koh², and \\ Jin Won $\mathrm{Huh}^{2}$
}

${ }^{1}$ Division of Pulmonary, Allergy and Critical Care Medicine, Hallym University Dongtan Sacred Heart Hospital, Hwaseong; ${ }^{2}$ Department of Pulmonary and Critical Care Medicine, Asan Medical Center, University of Ulsan College of Medicine, Seoul, Korea

Received: May 5, 2019

Revised : August 1, 2019

Accepted: August 9, 2019

Correspondence to

Jin Won Huh, M.D.

Department of Pulmonary and Critical Care Medicine, Asan Medical Center, University of Ulsan College of Medicine, 88 Olympic-ro 43-gil, Songpa-gu, Seoul 05505, Korea

Tel: +82-2-3010-3985

Fax: $+82-2-3010-6968$

E-mail:jwhuh@amc.seoul.kr https://orcid.org/0000-00023449-0461
Background/Aims: It is unclear whether corticosteroid use in patients with acute respiratory distress syndrome (ARDS) improves survival. This study aimed to investigate whether the administration of corticosteroids to patients in the early phase of moderate to severe ARDS is associated with improved outcomes.

Methods: We analyzed the data of patients who received corticosteroids within 7 days of the onset of ARDS between June 2006 and December 2015 at a single tertiary teaching hospital. A total of 565 patients admitted with moderate to severe ARDS were eligible. The outcomes of patients treated with methylprednisolone 40 to 180 $\mathrm{mg} /$ day or equivalent $(\mathrm{n}=404)$ were compared to those who did not receive steroids $(n=161)$. The primary and secondary outcomes were 28 - and 90-day mortality rates, respectively. Propensity scores were used to adjust for baseline covariates.

Results: The overall mortality at 28 days was not significantly different between the corticosteroid-treated and control groups ( $43.8 \%$ vs. $41 \%, p=0.541$ ). At 90 days, the overall mortality rate was higher in the corticosteroid-treated group than in the control group $(59.2 \%$ vs. $48.4 \%, p=0.021)$. However, on propensity score matching, corticosteroid therapy was not associated with a higher 28 -day mortality rate (odds ratio, 1.031; 95\% confidence interval, 0.657 to $1.618 ; p=0.895$ ) and 90 days (odds ratio, 1.435; 95\% confidence interval, 0.877 to $2.348 ; p=0.151$ ).

Conclusions: Corticosteroid therapy was not associated with 28 - or 90-day mortality in the early phase of moderate to severe ARDS on propensity score matching analysis.

Keywords: Respiratory distress syndrome, adult; Corticosteroid; Mortality

\section{INTRODUCTION}

Acute respiratory distress syndrome (ARDS) is a type of acute hypoxemic respiratory failure characterized by pulmonary edema due to increased permeability of the pulmonary capillary endothelial and alveolar epithelial cells $[1,2]$. Diffuse alveolar damage, which occurs in the acute or exudative phase of ARDS, disrupts barrier func- tion in the lung, severely impairing gas exchange and compromising lung compliance [3]. Acute pulmonary inflammation, which involves various types of inflammatory cells and mediators, may be responsible for the pathogenesis of ARDS [4]. Corticosteroids, which feature potent anti-inflammatory and anti-fibrotic effects, may be beneficial in the treatment of ARDS.

However, it is unclear whether the use of corticoste- 
roids improves survival; additionally, the drug, dose, timing, and therapeutic duration with corticosteroids remain uncertain. Previous randomized trials failed to show convincing evidence of a reduced mortality rate with corticosteroid use in ARDS [5-8]. However, meta-analyses suggest that the use of low-dose corticosteroids may be associated with reduced mortality and that the early administration of low-dose corticosteroids may improve survival in patients with ARDS $[9,10]$. The ARDSnet study demonstrated that the administration of corticosteroids for more than 14 days after the onset of ARDS is associated with increased mortality [6]. Takaki et al. [11] recently reported that initial high-dose corticosteroid therapy followed by a tapering regimen (vs. a low-dose regimen) might worsen prognosis in ARDS. A nationwide Japanese study showed that the mortality rate was higher with methylprednisolone at doses greater than $500 \mathrm{mg} /$ day compared to the low-dose corticosteroid therapy [12].

It remains unclear whether the administration of low to moderate doses of corticosteroids in patients with ARDS is associated with improved outcomes. Therefore, we aimed to investigate the short- and long-term mortality rates associated with corticosteroid use in early ARDS and identify the factors associated with mortality.

\section{METHODS}

\section{Study design and eligible patients}

This retrospective study was conducted in the medical intensive care unit of Asan Medical Center, a 2,70o-bed tertiary referral hospital in Seoul, Korea. We included patients aged 18 years or older meeting ARDS criteria according to the Berlin definition [13]. Data on patients with ARDS who received corticosteroids within 7 days of the onset of ARDS between June 2006 and December 2015 were collected. Patients were categorized into the corticosteroid-treated group or the control group.

Patients were excluded if they met any of the following criteria: (1) initiation of corticosteroid therapy prior to the onset of ARDS; (2) initiation of corticosteroid therapy more than 7 days after the onset of ARDS; (3) single-dose therapy or less than $40 \mathrm{mg} /$ day of methylprednisolone or equivalent; (4) treatment with a dose greater than $180 \mathrm{mg} /$ day of methylprednisolone or equivalent; or (5) medical history indicating interstitial lung disease. The Institutional Review Board of Asan Medical Center approved this study and granted a waiver of consent given its retrospective design (approval No.: 2015-0164).

\section{Data collection and definitions}

At the time of intensive care unit (ICU) admission, data on age, sex, body mass index, Sequential Organ Failure Assessment (SOFA) score, underlying disease, partial pressure of oxygen/fraction of inspired oxygen $\left(\mathrm{PaO}_{2} /\right.$ $\mathrm{FiO}_{2}$ ) ratio, and cause of ARDS were collected. $\mathrm{PaO}_{2} /$ $\mathrm{FiO}_{2}$ ratio, radiologic findings, SOFA score, and clinical course were reanalyzed for the diagnosis of ARDS and sepsis based on the Berlin definition and sepsis-3 criteria. The timing of treatment initiation and duration were analyzed, and the corticosteroid dose was converted to the methylprednisolone equivalent. Treatment in the "early" phase was defined as initiating methylprednisolone or equivalent within 7 days of the onset of ARDS. The corticosteroid dose was defined as methylprednisolone 40 to $180 \mathrm{mg}$ comparable to 1 to $2 \mathrm{mg} / \mathrm{kg}$ of methylprednisolone as used in previous studies $[6,7]$. In this study, 25 patients who received a methylprednisolone dosage of 240 to 1,000 mg were excluded. Data from routine blood investigations including white blood cell count, platelet count, serum albumin and C-reactive protein levels, arterial blood gas analysis results, and lactic acid levels were collected. ARDS was classified as being of pulmonary or extra-pulmonary origin based on etiology. Pneumonia was defined as the appearance of a new lung infiltrate on the chest radiograph with clinical evidence suggestive of an infectious origin, including new onset fever, purulent sputum, leukocytosis, and decreased oxygenation. Hospital-associated pneumonia (HAP) was defined as pneumonia not incubating at the time of hospital admission and occurring 48 hours or more after admission. Ventilator-associated pneumonia (VAP) was defined as pneumonia occurring more than 48 hours after endotracheal intubation [14]. Patients with healthcare-associated pneumonia (HCAP) included those who were hospitalized in an acute care hospital for 2 or more days within 90 days of the infection; resided in a nursing home or long-term care facility; received recent intravenous antibiotic therapy, chemotherapy, or wound care within 30 days prior to the current infection; or attended a hospital or hemodialysis clinic [15]. Sepsis 
and septic shock were defined according to the sepsis-3 definition [16]. The above diagnoses were established comprehensively based on medical history, blood investigations, and imaging findings by electronic medical record review. The primary and secondary outcomes were 28 - and 90-day mortality rates, respectively.

\section{Statistical analysis}

Continuous variables are presented as median (interquartile range $[\mathrm{IQR}]$ ) or mean $\pm \mathrm{SD}$. Categorical variables are presented as number (percentage). The chi-square test or Fisher's exact test was used to compare categorical variables, while Student's $t$ test or the Mann-Whitney $U$ test was used to compare continuous variables as appropriate. Uni- and multivariate logistic regressions using the backward elimination method were performed to identify factors associated with 28- and 90-day mortality. Multivariate analysis was performed using variables with a $p$ value less than 0.2 on univariate analysis. The model calibration was evaluated using the Hosmer-Lemeshow goodness-of-fit test. The results are summarized as adjusted odds ratio (OR) and 95\% confidence interval (CI). All statistical analyses were two-sided, and $p$ values less than 0.05 were considered statistically significant. All analyses were performed using SPSS Statistics version 22.o (IBM Corp., Armonk, NY, USA).

Corticosteroids were administered to patients with hematologic disease, post-transplantation state, and pulmonary ARDS, suggesting a discrepancy in baseline disease severity between patients who received and those who did not receive corticosteroids. A propensity score (PS) analysis was performed to account for the intergroup differences in baseline characteristics and disease severity (Supplementary Table 1).

\section{RESULTS}

During the study period, 1,306 ICU patients met the Berlin definition of ARDS. Among 565 patients with moderate to severe ARDS, those treated with corticosteroids ( $n=404)$ were compared to those who were not treated with corticosteroids $(n=161)$ (Fig. 1). Commonly used corticosteroids included hydrocortisone (253 patients [62.6\%]), methylprednisolone (141 patients [34.9\%]), dexamethasone (eight patients [2.0\%]), and predniso-

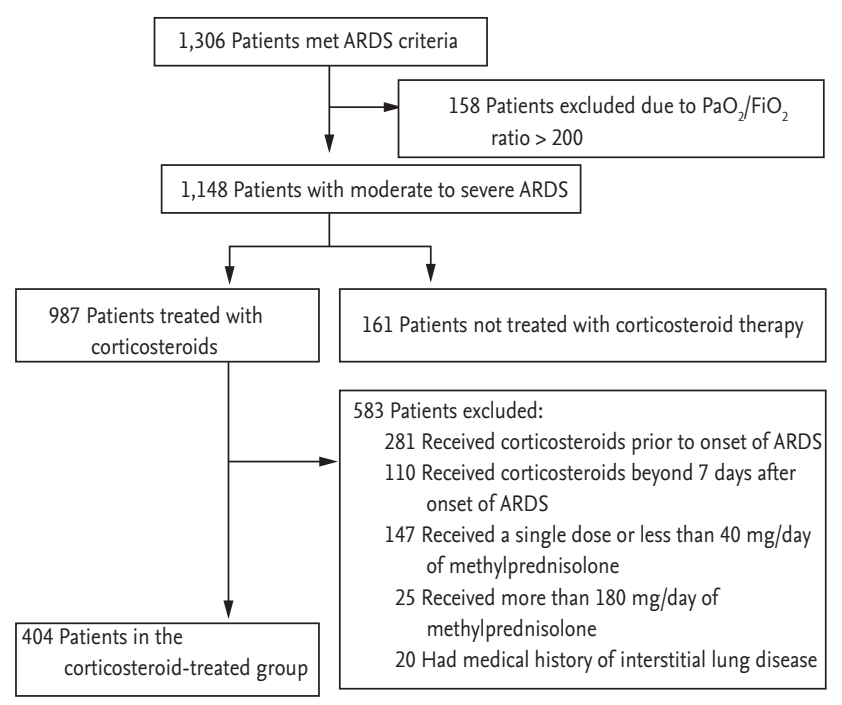

Figure 1. Flow chart of the patients. ARDS, acute respiratory distress syndrome; $\mathrm{FiO}_{2}$, fraction of inspired oxygen; $\mathrm{PaO}_{2}$, arterial partial pressure of oxygen.

lone (two patients [0.5\%]) (Supplementary Table 2). The median duration of corticosteroid use was 13 days (IQR, 6 to 29 days). Pneumonia (61.4\%), sepsis (15.8\%), and aspiration (9.9\%) were the most common causes of ARDS. The median $\mathrm{PaO}_{2} / \mathrm{FiO}_{2}$ ratio was 100 (IQR, 71 to 136 ), while the mean SOFA score at ICU admission was $11 \pm$ 4. There were no significant intergroup differences in SOFA score or $\mathrm{PaO}_{2} / \mathrm{FiO}_{2}$ ratio (Table 1).

The outcomes of the patients with ARDS are shown in Table 2. The overall 28-day mortality rate was $43.0 \%$, while the 90-day mortality rate was $56.1 \%$. The mortality rate at 28 days did not differ significantly between the corticosteroid-treated and control groups (43.8\% vs. $41.0 \%, p=0.541)$. However, the mortality rate at 90 days was higher in the corticosteroid-treated group than in the control group $(59.2 \%$ vs. $48.4 \%, p=0.021)$. Length of ICU stay was longer in the corticosteroid-treated group than in the control group ( 16 days vs. 10 days, $p<$ $0.001)$. The mean mechanical ventilation-free days until day 28 was longer in the control group than in the corticosteroid-treated group $(9.7 \pm 10.5$ days vs. $7.3 \pm 9.1$ days, $p=0.013$ ). The rate of bacteremia was significantly higher in the corticosteroid-treated group than in the control group ( $41.1 \%$ vs. $30.4 \%, p=0.019$ ). There was no significant intergroup difference in the incidence of 
Table 1. Patients' baseline characteristics

\begin{tabular}{|c|c|c|c|c|}
\hline Characteristic & Total $(n=565)$ & Control (n = 161) & Corticosteroid-treated $(n=404)$ & $p$ value \\
\hline Age, yr & $63 \pm 13$ & $63 \pm 15$ & $63 \pm 13$ & 0.932 \\
\hline Male sex & $379(67.1)$ & $122(75.8)$ & $257(63.6)$ & 0.006 \\
\hline SOFA score & $11 \pm 4$ & $11 \pm 4$ & $11 \pm 4$ & 0.416 \\
\hline Body mass index, $\mathrm{kg} / \mathrm{m}^{2}$ & $22.6 \pm 4.0$ & $22.8 \pm 4.6$ & $22.5 \pm 3.7$ & 0.559 \\
\hline Glasgow coma score & $3(2-9)$ & $4(2-9)$ & $2(2-9)$ & 0.416 \\
\hline $\mathrm{PaO}_{2} / \mathrm{FiO}_{2}$ ratio & $100(71-136)$ & $105(77-141)$ & $97(67-133)$ & 0.065 \\
\hline \multicolumn{5}{|l|}{ Comorbidity } \\
\hline Diabetes mellitus & $182(32.2)$ & $60(37 \cdot 3)$ & $122(30.2)$ & 0.105 \\
\hline Solid tumor & $169(29.9)$ & $58(36.0)$ & $111(27 \cdot 5)$ & 0.045 \\
\hline Cardiovascular disease & $38(6.7)$ & $12(7 \cdot 5)$ & $26(6.4)$ & 0.663 \\
\hline Chronic pulmonary disease & $77(13.6)$ & $19(11.8)$ & $58(14 \cdot 4)$ & 0.424 \\
\hline Chronic kidney disease & $45(8.0)$ & $12(7 \cdot 5)$ & $33(8.2)$ & 0.777 \\
\hline Cerebrovascular disease & $43(7 \cdot 6)$ & $17(10.6)$ & $26(6.4)$ & 0.095 \\
\hline Hematologic disease & $73(12.9)$ & $11(6.8)$ & $62(15 \cdot 4)$ & 0.007 \\
\hline Chronic liver disease & $73(12.9)$ & $30(18.6)$ & $43(10.6)$ & 0.011 \\
\hline Post-transplant & $49(8.7)$ & $4(2.5)$ & $45(11)$ & 0.001 \\
\hline \multicolumn{5}{|l|}{ Cause of ARDS } \\
\hline CAP & $100(17.7)$ & $26(16.2)$ & $74(18.3)$ & 0.542 \\
\hline HCAP & $147(26.0)$ & $28(17 \cdot 4)$ & $119(29 \cdot 5)$ & 0.003 \\
\hline HAP & $100(17.7)$ & $32(19.9)$ & $68(16.8)$ & 0.392 \\
\hline Aspiration & $56(9.9)$ & $23(14 \cdot 3)$ & $33(8.2)$ & 0.028 \\
\hline Other $^{\mathrm{a}}$ & $35(6.2)$ & $5(3.1)$ & $30(7 \cdot 4)$ & 0.055 \\
\hline Sepsis & $89(15.8)$ & $27(16.8)$ & $62(15 \cdot 4)$ & 0.675 \\
\hline Non-sepsis ${ }^{\mathrm{b}}$ & $31(5 \cdot 5)$ & $14(8.7)$ & $17(4.2)$ & 0.035 \\
\hline Pulmonary cause & $434(76.8)$ & $112(69.6)$ & $322(79 \cdot 7)$ & 0.010 \\
\hline Extrapulmonary cause & $117(20.7)$ & $41(25 \cdot 5)$ & $76(18.8)$ & 0.078 \\
\hline
\end{tabular}

Values are presented as mean $\pm \mathrm{SD}$, number (\%), or median (interquartile range).

SOFA, sequential organ failure assessment; $\mathrm{PaO}_{2}$, arterial partial pressure of oxygen; $\mathrm{FiO}_{2}$, fraction of inspired oxygen; ARDS, acute respiratory distress syndrome; CAP, community-acquired pneumonia; HCAP, healthcare-associated pneumonia; HAP, hospital-acquired pneumonia.

${ }^{a}$ Other cause of ARDS included drowning $(n=3)$, inhalation $(n=2)$, vasculitis $(n=4)$, diffuse alveolar hemorrhage $(n=14)$, and drug-induced $(n=12)$.

${ }^{\mathrm{b}}$ Non-sepsis included pancreatitis $(\mathrm{n}=1)$, transfusion-related acute lung injury $(\mathrm{n}=23)$, trauma $(\mathrm{n}=6)$, and anaphylaxis $(\mathrm{n}=1)$.

Clostridium difficile infection and VAP. The incidence of pneumothorax was higher in the corticosteroid-treated group (10.9\% vs. $3.7 \%, p=0.007$ ).

On multivariate analysis, the SOFA score (OR, 1.143; $95 \%$ CI, 1.086 to 1.206; $p<0.001)$, presence of solid tumor (OR, 2.102; 95\% CI, 1.430 to 3.105; $p<0.001$ ), chronic liver disease (OR, 2.389; 95\% CI, 1.370 to $4.247 ; p=0.003$ ), and hematologic disease (OR, 1.982; 95\% CI, 1.176 to 3.371; $p$
$=0.011$ ) were significantly associated with 28 -day mortality (Table 3). The 90-day mortality rate was associated with corticosteroid therapy (OR, 1.794; 95\% CI, 1.199 to $2.695 ; p=0.005)$, SOFA score (OR, 1.121; $95 \% \mathrm{CI}, 1.065$ to 1.182; $p<0.001)$, presence of solid tumor (OR, 2.533; 95\% CI, 1.696 to $3.825 ; p<0.001)$, chronic liver disease (OR, 2.228; 95\% CI, 1.269 to $4.268 ; p=0.007$ ), hematologic disease (OR, 3.068; 95\% CI, 1.710 to 5.747; $p<0.001$ ), and 
Table 2. Outcomes of patients with acute respiratory distress syndrome

\begin{tabular}{lccc}
\hline Variable & Control $(\mathrm{n}=161)$ & Corticosteroid-treated $(\mathrm{n}=404)$ & $p$ value \\
\hline 28-Day mortality & $66(41.0)$ & $177(43.8)$ & 0.541 \\
90-Day mortality & $78(48.4)$ & $239(59.2)$ & 0.021 \\
No. of ventilator-free days at day 28 & $9.7 \pm 10.5$ & $7.3 \pm 9.1$ & 0.013 \\
Tracheostomy & $49(30.4)$ & $158(39.1)$ & 0.053 \\
Hospital stay, day & $23(14-45)$ & $28(15-52)$ & 0.121 \\
ICU stay, day & $10(5-19)$ & $16(8-28)$ & $<0.001$ \\
\hline Duration of mechanical ventilation & $8(4-12)$ & $12(7-18)$ & $<0.001$ \\
Bacteremia & $49(30.4)$ & $166(41.1)$ & 0.019 \\
Clostridium difficile infection & $5(3.1)$ & $18(4.5)$ & 0.464 \\
Ventilator-associated pneumonia & $5(3.1)$ & $29(7.2)$ & 0.066 \\
Pneumothorax & $6(3.7)$ & $44(10.9)$ & 0.007 \\
\hline
\end{tabular}

Values are presented as number (\%), mean $\pm \mathrm{SD}$, or median (interquartile range).

ICU, intensive care unit.

Table 3. Factors associated with 28 -day mortality

\begin{tabular}{|c|c|c|c|c|}
\hline \multirow{2}{*}{ Variable } & \multicolumn{2}{|c|}{ Univariate analysis } & \multicolumn{2}{|c|}{ Multivariate analysis } \\
\hline & OR $(95 \% \mathrm{CI})$ & $p$ value & OR $(95 \% \mathrm{CI})$ & $p$ value \\
\hline Age, yr & $1.000(0.988-1.013)$ & 0.952 & & \\
\hline Male sex & $1.177(0.827-1.675)$ & 0.365 & & \\
\hline SOFA score & $1.164(1.108-1.222)$ & $<0.001$ & $1.143(1.086-1.206)$ & $<0.001$ \\
\hline Body mass index & $0.991(0.951-1.034)$ & 0.684 & & \\
\hline Glasgow Coma Scale score & $0.967(0.934-1.001)$ & 0.060 & & \\
\hline Corticosteroid use & $1.116(0.771-1.616)$ & 0.560 & $1.176(0.788-1.762)$ & 0.430 \\
\hline Diabetes mellitus & $0.992(0.695-1.416)$ & 0.965 & & \\
\hline Solid tumor & $1.753(1.219-2.520)$ & 0.002 & $2.102(1.430-3.105)$ & $<0.001$ \\
\hline Cardiovascular disease & $0.656(0.328-1.311)$ & 0.233 & & \\
\hline Chronic pulmonary disease & $0.755(0.460-1.237)$ & 0.264 & & \\
\hline Chronic kidney disease & $0.854(0.459-1.589)$ & 0.618 & & \\
\hline Cerebrovascular disease & $0.537(0.274-1.054)$ & 0.071 & & \\
\hline Hematologic disease & $1.913(1.163-3.146)$ & 0.011 & $1.982(1.176-3.371)$ & 0.011 \\
\hline Chronic liver disease & $3.057(1.817-5.145)$ & $<0.001$ & $2.389(1.370-4.247)$ & 0.003 \\
\hline Post-transplant & $0.733(0.400-1.345)$ & 0.316 & & \\
\hline CAP & $0.441(0.275-0.707)$ & 0.001 & & \\
\hline HCAP & $1.204(0.826-1.757)$ & 0.334 & & \\
\hline HAP & $1.129(0.731-1,742)$ & 0.585 & & \\
\hline Aspiration & $1.138(0.654-1.981)$ & 0.646 & & \\
\hline Other & $0.575(0.276-1.199)$ & 0.140 & & \\
\hline Sepsis & $1.934(1.223-3.058)$ & 0.005 & & \\
\hline Non-sepsis & $0.700(0.329-1.489)$ & 0.354 & & \\
\hline
\end{tabular}

OR, odds ratio; CI, confidence interval; SOFA, Sequential Organ Failure Assessment; CAP, community-acquired pneumonia; HCAP, healthcare-associated pneumonia; HAP, hospital-acquired pneumonia. 
Table 4. Factors associated with 90-day mortality

\begin{tabular}{|c|c|c|c|c|}
\hline \multirow{2}{*}{ Variable } & \multicolumn{2}{|c|}{ Univariate analysis } & \multicolumn{2}{|c|}{ Multivariate analysis } \\
\hline & OR $(95 \% \mathrm{CI})$ & $p$ value & OR $(95 \% \mathrm{CI})$ & $p$ value \\
\hline Age, yr & $1.009(0.997-1.022)$ & 0.139 & & \\
\hline Sex, male & $0.988(0.694-1.408)$ & 0.949 & & \\
\hline SOFA score & $1.136(1.083-1.191)$ & $<0.001$ & $1.121(1.065-1.182)$ & $<0.001$ \\
\hline Body mass index & $0.982(0.942-1.024)$ & 0.392 & & \\
\hline Glasgow Coma Scale score & $0.956(0.924-0.989)$ & 0.009 & & \\
\hline Corticosteroid use & $1.541(1.068-2.225)$ & 0.021 & $1.794(1.199-2.695)$ & 0.005 \\
\hline Diabetes mellitus & $0.846(0.593-1.206)$ & 0.354 & & \\
\hline Solid tumor & $2.123(1.452-3.105)$ & $<0.001$ & $2.533(1.696-3.825)$ & $<0.001$ \\
\hline Cardiovascular disease & $0.860(0.445-1.664)$ & 0.655 & & \\
\hline Chronic pulmonary disease & $0.687(0.424-1.112)$ & 0.127 & & \\
\hline Chronic kidney disease & $0.601(0.325-1.109)$ & 0.103 & & \\
\hline Cerebrovascular disease & $0.659(0.353-1.229)$ & 0.190 & & \\
\hline Hematologic disease & $2.682(1.531-4.700)$ & 0.001 & $3.068(1.710-5.747)$ & $<0.001$ \\
\hline Chronic liver disease & $2.475(1.425-4.298)$ & 0.001 & $2.288(1.269-4.268)$ & 0.007 \\
\hline Post-transplant & $0.610(0.339-1.100)$ & 0.101 & $0.429(0.222-0.816)$ & 0.011 \\
\hline CAP & $0.500(0.323-0.775)$ & 0.002 & & \\
\hline HCAP & $1.231(0.840-1.804)$ & 0.286 & & \\
\hline HAP & $1.277(0.821-1.986)$ & 0.278 & & \\
\hline Aspiration & $1.234(0.702-2.169)$ & 0.465 & & \\
\hline Other & $0.641(0.322-1.273)$ & 0.204 & & \\
\hline Sepsis & $1.763(1.093-2.845)$ & 0.020 & & \\
\hline Non-sepsis & $0.410(0.193-0.872)$ & 0.021 & & \\
\hline
\end{tabular}

OR, odds ratio; CI, confidence interval; SOFA, Sequential Organ Failure Assessment; CAP, community-acquired pneumonia; HCAP, healthcare-associated pneumonia; HAP, hospital-acquired pneumonia.

\section{Table 5. Analysis of patient outcomes}

\begin{tabular}{lcc}
\hline Variable & OR $(95 \% \mathrm{CI})$ & p value \\
\hline 28-Day mortality & & 0.560 \\
Univariate adjusted & $1.116(0.771-1.616)$ & 0.430 \\
Multivariate adjusted & $1.176(0.788-1.762)$ & 0.895 \\
Propensity score matched & $1.031(0.657-1.618)$ & 0.021 \\
$90-D a y$ mortality & & 0.005 \\
Univariate adjusted & $1.541(1.068-2.225)$ & 0.151 \\
Multivariate adjusted & $1.794(1.199-2.695)$ & $1.435(0.877-2.348)$ \\
Propensity score matched & & \\
\hline
\end{tabular}

OR, odds ratio; CI, confidence interval.

post-transplant status (OR, 0.429; 95\% CI, 0.222 to 0.816 ; $p=0.011$ ) (Table 4).

Propensity-matched analysis (Table 5) showed that corticosteroid therapy was not associated with 28-day (OR, 1.031; 95\% CI, 0.657 to $1.618 ; p=0.895$ ) or 90-day mortality (OR, 1.435; 95\% CI, 0.877 to 2.348 ; $p=0.151$ ). 


\section{DISCUSSION}

Here we investigated the effect of corticosteroid therapy on survival in the early phase of ARDS. We could not demonstrate an association between corticosteroid therapy and short-term mortality; on the contrary, it increased the odds of longer-term mortality. Besides, the incidence of complications including bacteremia and pneumothorax was higher in patients treated with corticosteroids. According to our results, corticosteroid use was not associated with 90-day mortality after PS matching.

In this study, we aimed to identify the efficacy of corticosteroids in the early phase of ARDS. However, there were significant differences in the baseline characteristics between the corticosteroid-treated and control groups. The corticosteroid-treated patients had a higher incidence of hematologic disease, post-transplantation state, and pulmonary ARDS. The median $\mathrm{PaO}_{2} / \mathrm{FiO}_{2}$ ratio tended to be lower in patients treated with corticosteroids. Corticosteroid use in patients with ARDS did not affect survival after PS matching. The higher longer-term mortality in the corticosteroid-treated group may be associated with the underlying disease, such as hematologic malignancy, or post-transplantation state.

Corticosteroid use at 3 to 7 days after the onset of ARDS reportedly improves survival [7,9,17-19]. Meduri et al. [7] emphasized the importance of the downregulation of systemic inflammation in the early phase of ARDS. Lee et al. [17] suggested that fibroproliferation is an early response to lung injury and is inhibited by early low-dose steroid therapy. According to a previous report on the histological stages of ARDS based on autopsy findings, proliferative changes were noted in only $54 \%$ of patients with ARDS of less than 1-week duration [20]. Therefore, it is difficult to explain the beneficial effect of corticosteroids in the early phase of ARDS with heterogeneity.

One potential adverse effect of corticosteroid therapy is hospital-acquired infections. Previous studies demonstrated that low-dose corticosteroids do not increase the incidence of infectious complications $[6,7]$. However, Peter et al. [21] reported a trend toward an increased risk of infection with increasing corticosteroid dose. In a meta-analysis, Ruan et al. [22] found that corticosteroids tended to decrease the infection risk in randomized controlled studies (risk ratio, 0.83; 95\% CI, 0.65 to 1.06) but they increase the infection risk in cohort studies (risk ratio, 1.35; 95\% CI, 0.99 to 1.84). We noted a higher incidence of bacteremia in the corticosteroid-treated group. These observations indicate that the use of corticosteroids could be associated with a higher incidence of infective complications. Furthermore, prolonged ICU stays and ventilation duration were observed in the corticosteroid-treated group. Ventilation duration is thought to increase the likelihood of barotrauma [23] and was longer in the corticosteroid-treated group than in the control group (12 days vs. 8 days, $p<0.001$ ). The higher incidence of pneumothorax may have contributed to the longer ICU stay [24].

There are several clinical guidelines for the management of ARDS with conflicting recommendations [2528]. Korean and Scandinavian guidelines recommended against routine corticosteroid use because there was no significant survival benefit of corticosteroids $[25,26]$. In the Japanese guideline, corticosteroid use was suggested because the corticosteroid therapy was associated with a decreased duration of mechanical ventilation and did not significantly increase the infection rate [28]. However, as shown in our study results, concern about infectious complications of corticosteroid therapy continues. As highlighted in the Scandinavian guideline, corticosteroid therapy should target subgroups in which corticosteroids are indicated. Therefore, further studies to identify subgroups that are likely to respond to corticosteroids are needed.

Our study has several limitations. First, it was not a randomized controlled trial, thus there were baseline intergroup discrepancies in patient characteristics and disease severity. The corticosteroid-treated group had a higher proportion of female patients, hematologic disease, post-transplantation status, and pulmonary cause than the control group. Patients with more serious illnesses might have been included in the corticosteroid-treated group, although we performed PS matching to minimize these baseline differences. Second, this is a retrospective study with a 10-year study period. The choice of drug or treatment duration was not standardized. Clinicians determined the treatment based on the clinical course of patients based on the Meduri protocol [8] or Surviving Sepsis Campaign Guidelines [29]. Third, the long study period enabled the enrollment of more patients, while changes in treatment protocol, 
such as prone positioning and neuromuscular blocking agents might affect survival. Fourth, we did not present data on the incidence of ICU-acquired weakness, hyperglycemia, and mechanical ventilation parameters. Nonetheless, the strengths of this study include its large sample size with the diagnosis of ARDS based on the Berlin definition and evaluation of the effect of low to moderate doses of corticosteroids in the early phase of ARDS. Considering the conflicting evidence from previous studies, there may be subgroups of ARDS patients in whom corticosteroid treatment may improve outcomes. Therefore, caution should be exercised while considering corticosteroid therapy for ARDS patients until these subgroups are identified. In addition, a prospective multicenter study is needed to provide more convincing evidence of the effect of corticosteroid in ARDS.

In conclusion, here we observed no survival benefit with corticosteroid therapy in the early phase of moderate to severe ARDS; on the contrary, it may be associated with a higher incidence of 9o-day mortality. Adjunctive corticosteroids should be carefully considered in the treatment of ARDS.

\section{KEY MESSAGE}

1. There was no survival benefit of corticosteroid therapy in the early phase of moderate to severe acute respiratory distress syndrome.

2. The incidence of complications, including bacteremia and pneumothorax was higher in patients treated with corticosteroids.

\section{Conflict of interest}

No potential conflict of interest relevant to this article was reported.

\section{REFERENCES}

1. Koh Y. Update in acute respiratory distress syndrome. J Intensive Care 2014;2:2.

2. Matthay MA, Ware LB, Zimmerman GA. The acute respiratory distress syndrome. J Clin Invest 2012;122:2731-2740.

3. Piantadosi CA, Schwartz DA. The acute respiratory distress syndrome. Ann Intern Med 2004;141:460-470.
4. Bernard GR, Artigas A, Brigham KL, et al. The American-European Consensus Conference on ARDS: definitions, mechanisms, relevant outcomes, and clinical trial coordination. Am J Respir Crit Care Med 1994;149(3 Pt 1):818-824.

5. Bernard GR, Luce JM, Sprung CL, et al. High-dose corticosteroids in patients with the adult respiratory distress syndrome. N Engl J Med 1987;317:1565-1570.

6. Steinberg KP, Hudson LD, Goodman RB, et al. Efficacy and safety of corticosteroids for persistent acute respiratory distress syndrome. N Engl J Med 2006;354:1671-1684.

7. Meduri GU, Golden E, Freire AX, et al. Methylprednisolone infusion in early severe ARDS: results of a randomized controlled trial. Chest 2007;131:954-963.

8. Meduri GU, Headley AS, Golden E, et al. Effect of prolonged methylprednisolone therapy in unresolving acute respiratory distress syndrome: a randomized controlled trial. JAMA 1998;280:159-165.

9. Yang ZG, Lei XL, Li XL. Early application of low-dose glucocorticoid improves acute respiratory distress syndrome: a meta-analysis of randomized controlled trials. Exp Ther Med 2017;13:1215-1224.

10. Tang BM, Craig JC, Eslick GD, Seppelt I, McLean AS. Use of corticosteroids in acute lung injury and acute respiratory distress syndrome: a systematic review and meta-analysis. Crit Care Med 2009;37:1594-1603.

11. Takaki M, Ichikado K, Kawamura K, Gushima Y, Suga M. The negative effect of initial high-dose methylprednisolone and tapering regimen for acute respiratory distress syndrome: a retrospective propensity matched cohort study. Crit Care 2017;21:135.

12. Kido T, Muramatsu K, Asakawa T, et al. The relationship between high-dose corticosteroid treatment and mortality in acute respiratory distress syndrome: a retrospective and observational study using a nationwide administrative database in Japan. BMC Pulm Med 2018;18:28.

13. ARDS Definition Task Force, Ranieri VM, Rubenfeld GD, et al. Acute respiratory distress syndrome: the Berlin Definition. JAMA 2012;307:2526-2533.

14. Kalil AC, Metersky ML, Klompas M, et al. Management of adults with hospital-acquired and ventilator-associated pneumonia: 2016 Clinical Practice Guidelines by the Infectious Diseases Society of America and the American Thoracic Society. Clin Infect Dis 2016;63:e61-e111.

15. American Thoracic Society; Infectious Diseases Society of America. Guidelines for the management of adults 
with hospital-acquired, ventilator-associated, and healthcare-associated pneumonia. Am J Respir Crit Care Med 2005;171:388-416.

16. Singer M, Deutschman CS, Seymour CW, et al. The third international consensus definitions for sepsis and septic shock (sepsis-3). JAMA 2016;315:801-810.

17. Lee HS, Lee JM, Kim MS, Kim HY, Hwangbo B, Zo JI. Low-dose steroid therapy at an early phase of postoperative acute respiratory distress syndrome. Ann Thorac Surg 2005;79:405-410.

18. Meduri GU, Bridges L, Shih MC, Marik PE, Siemieniuk RAC, Kocak M. Prolonged glucocorticoid treatment is associated with improved ARDS outcomes: analysis of individual patients' data from four randomized trials and trial-level meta-analysis of the updated literature. Intensive Care Med 2016;42:829-840.

19. Agarwal R, Nath A, Aggarwal AN, Gupta D. Do glucocorticoids decrease mortality in acute respiratory distress syndrome? A meta-analysis. Respirology 2007;12:585-590.

20. Thille AW, Esteban A, Fernandez-Segoviano P, et al. Chronology of histological lesions in acute respiratory distress syndrome with diffuse alveolar damage: a prospective cohort study of clinical autopsies. Lancet Respir Med 2013;1:395-401.

21. Peter JV, John P, Graham PL, Moran JL, George IA, Bersten A. Corticosteroids in the prevention and treatment of acute respiratory distress syndrome (ARDS) in adults: meta-analysis. BMJ 2008;336:1006-1009.

22. Ruan SY, Lin HH, Huang CT, Kuo PH, Wu HD, Yu CJ. Ex- ploring the heterogeneity of effects of corticosteroids on acute respiratory distress syndrome: a systematic review and meta-analysis. Crit Care 2014;18:R63.

23. Cullen DJ, Caldera DL. The incidence of ventilator-induced pulmonary barotrauma in critically ill patients. Anesthesiology 1979;50:185-190.

24. De Lassence A, Timsit JF, Tafflet M, et al. Pneumothorax in the intensive care unit: incidence, risk factors, and outcome. Anesthesiology 2006;104:5-13.

25. Cho YJ, Moon JY, Shin ES, et al. Clinical practice guideline of acute respiratory distress syndrome. Tuberc Respir Dis (Seoul) 2016;79:214-233.

26. Claesson J, Freundlich M, Gunnarsson I, et al. Scandinavian clinical practice guideline on fluid and drug therapy in adults with acute respiratory distress syndrome. Acta Anaesthesiol Scand 2016;60:697-709.

27. Annane D, Pastores SM, Rochwerg B, et al. Guidelines for the diagnosis and management of critical illness-related corticosteroid insufficiency (CIRCI) in critically ill patients (part I): Society of Critical Care Medicine (SCCM) and European Society of Intensive Care Medicine (ESICM) 2017. Intensive Care Med 2017;43:1751-1763.

28. Hashimoto S, Sanui M, Egi M, et al. The clinical practice guideline for the management of ARDS in Japan. J Intensive Care 2017;5:50.

29. Rhodes A, Evans LE, Alhazzani W, et al. Surviving sepsis campaign: international guidelines for management of sepsis and septic shock: 2016. Intensive Care Med 2017;43:304-377. 
Supplementary Table 1. Patient characteristics after propensity score matching

\begin{tabular}{|c|c|c|c|c|c|c|c|c|}
\hline \multirow{2}{*}{ Characteristic } & \multicolumn{5}{|c|}{ Total data $(\mathrm{n}=565)$} & \multicolumn{3}{|c|}{ Matched data $(\mathrm{n}=268)$} \\
\hline & Steroid & No $(n=161)$ & Yes $(n=404)$ & $p$ value & SDM & No $(n=134)$ & Yes $(n=134)$ & SDM \\
\hline Sex & & & & 0.006 & 0.267 & & & 0.052 \\
\hline Male & & $122(75.78)$ & $257(63.61)$ & & & $100(74.63)$ & $103(76.87)$ & \\
\hline Female & & $39(24.22)$ & $147(36.39)$ & & & $34(25 \cdot 37)$ & $31(3.13)$ & \\
\hline Age, yr & & $63.006 \pm 14.626$ & $63.114 \pm 12.957$ & 0.932 & 0.008 & $63.61 \pm 14.46$ & $63.77 \pm 12.88$ & 0.011 \\
\hline Body mass index, $\mathrm{kg} / \mathrm{m}^{2}$ & & $22.769 \pm 4.604$ & $22.529 \pm 3.730$ & 0.559 & 0.057 & $22.41 \pm 4.34$ & $22.40 \pm 3.57$ & 0.002 \\
\hline \multirow[t]{2}{*}{ Diabetes mellitus } & No & $101(62.73)$ & $282(69.8)$ & 0.105 & 0.150 & $87(64.93)$ & $84(62.69)$ & 0.047 \\
\hline & Yes & $60(37.27)$ & $122(30.2)$ & & & $47(35.07)$ & $50(37 \cdot 31)$ & \\
\hline \multirow[t]{2}{*}{ Solid tumor } & No & $103(63.98)$ & $293(72.52)$ & 0.045 & 0.184 & $89(66.42)$ & $88(65.67)$ & 0.016 \\
\hline & Yes & $58(36.02)$ & $111(27.48)$ & & & $45(33.58)$ & $46(34 \cdot 33)$ & \\
\hline \multirow[t]{2}{*}{ Cardiovascular disease } & No & $149(92.55)$ & $378(93.56)$ & 0.663 & 0.040 & $123(91.79)$ & $124(92.54)$ & 0.028 \\
\hline & Yes & $12(7.45)$ & $26(6.44)$ & & & $11(8.21)$ & $10(7.46)$ & \\
\hline \multirow[t]{2}{*}{ Pulmonary disease } & No & $142(88.2)$ & $346(85.64)$ & 0.424 & 0.076 & $116(86.57)$ & $118(88.06)$ & 0.045 \\
\hline & Yes & $19(11.8)$ & $58(14.36)$ & & & $18(13.43)$ & $16(11.94)$ & \\
\hline \multirow[t]{2}{*}{ Chronic kidney disease } & No & $149(92.55)$ & $371(91.83)$ & 0.777 & 0.027 & $123(91.79)$ & $122(91.04)$ & 0.027 \\
\hline & Yes & $12(7.45)$ & $33(8.17)$ & & & $11(8.21)$ & $12(8.96)$ & \\
\hline \multirow[t]{2}{*}{ Cerebrovascular disease } & No & $144(89.44)$ & $378(93.56)$ & 0.095 & 0.148 & $120(89.55)$ & $122(91.04)$ & 0.050 \\
\hline & Yes & $17(10.56)$ & $2(66.44)$ & & & $14(10.45)$ & $12(8.96)$ & \\
\hline \multirow[t]{2}{*}{ Hematologic disease } & No & $150(93.17)$ & $342(84.65)$ & 0.007 & 0.274 & $123(91.79)$ & $126(94.03)$ & 0.087 \\
\hline & Yes & $11(6.83)$ & $62(15 \cdot 35)$ & & & $11(8.21)$ & $8(5.97)$ & \\
\hline \multirow[t]{2}{*}{ Chronic liver disease } & No & $131(81.37)$ & $361(89.36)$ & 0.011 & 0.228 & $116(86.57)$ & $113(84 \cdot 33)$ & 0.064 \\
\hline & Yes & $30(18.63)$ & $43(10.64)$ & & & $18(13.43)$ & $21(15 \cdot 67)$ & \\
\hline \multirow[t]{2}{*}{ Post-transplant state } & No & $157(97.52)$ & $359(88.86)$ & 0.001 & 0.349 & $130(97.01)$ & $128(95 \cdot 52)$ & 0.079 \\
\hline & Yes & $4(2.48)$ & $45(11.14)$ & & & $4(2.99)$ & $6(4 \cdot 48)$ & \\
\hline GCS score & & $4(2-9)$ & $2(2-9)$ & 0.788 & 0.026 & $4(2-9)$ & $3(2-14)$ & 0.042 \\
\hline SOFA score & & $11.081 \pm 3.679$ & $11.366 \pm 3.798$ & 0.416 & 0.076 & $11.01 \pm 3.69$ & $10.93 \pm 3.63$ & 0.025 \\
\hline \multirow[t]{2}{*}{ Community-acquired pneumonia } & No & $135(83.85)$ & $330(81.68)$ & 0.542 & 0.057 & $110(82.09)$ & $112(83.58)$ & 0.040 \\
\hline & Yes & $26(16.15)$ & $74(18.32)$ & & & $24(17.91)$ & $22(16.42)$ & \\
\hline \multirow[t]{2}{*}{ Healthcare-associated pneumonia } & No & $133(82.61)$ & $285(70.54)$ & 0.003 & 0.288 & $111(82.84)$ & $111(82.84)$ & 0.000 \\
\hline & Yes & $28(17 \cdot 39)$ & $119(29.46)$ & & & $23(17.16)$ & $23(17.16)$ & \\
\hline \multirow[t]{2}{*}{ Hospital-acquired pneumonia } & No & $129(80.12)$ & $336(83.17)$ & 0.392 & 0.079 & $111(82.84)$ & $109(81.34)$ & 0.039 \\
\hline & Yes & $32(19.88)$ & $68(16.83)$ & & & $23(17.16)$ & $25(18.66)$ & \\
\hline \multirow[t]{2}{*}{ Aspiration } & No & $138(85.71)$ & $371(91.83)$ & 0.028 & 0.195 & $117(87.31)$ & $114(85.07)$ & 0.065 \\
\hline & Yes & $23(14.29)$ & $33(8.17)$ & & & $17(12.69)$ & $20(14.93)$ & \\
\hline \multirow[t]{2}{*}{ Others } & No & $156(96.89)$ & $374(92.57)$ & 0.055 & 0.194 & $129(96.27)$ & $128(95.52)$ & 0.038 \\
\hline & Yes & $5(3.11)$ & $30(7.43)$ & & & $5(3.73)$ & $6(4.48)$ & \\
\hline \multirow[t]{2}{*}{ Sepsis } & No & $134(83.23)$ & $342(84.65)$ & 0.675 & 0.039 & $109(81.34)$ & $107(79.85)$ & 0.038 \\
\hline & Yes & $27(16.77)$ & $62(15 \cdot 35)$ & & & $25(18.66)$ & $27(20.15)$ & \\
\hline \multirow[t]{2}{*}{ Non-sepsis } & No & $147(91.3)$ & $387(95.79)$ & 0.035 & 0.183 & $122(91.04)$ & $125(93.28)$ & 0.083 \\
\hline & Yes & $14(8.7)$ & $17(4.21)$ & & & $12(8.96)$ & $9(6.72)$ & \\
\hline
\end{tabular}

Values are presented as number (\%), mean \pm SD, or median (interquartile range). Categorical variables: chi-square test or Fisher's exact test (discrimination: c-statistics $=0.792$; calibration: Hosmer-Lemeshow statistics $p$ value $=0.1580$ ). Continuous variables: Student's t test.

SDM, standardized difference of the mean; GCS, Glasgow Coma Scale; SOFA, Sequential Organ Failure Assessment. 
Baek MS, et al. Corticosteroid in ARDS

Supplementary Table 2. Duration and dose of corticosteroids

\begin{tabular}{lccc}
\hline Corticosteroids & Total $(\mathrm{n}=404)$ & Equivalent dose, mg & Duration, day \\
\hline Methylprednisolone & $141(34.9)$ & $93 \pm 37$ & $31 \pm 29$ \\
Hydrocortisone & $253(62.6)$ & $44 \pm 8$ & $20 \pm 30$ \\
Dexamethasone & $8(2.0)$ & $122 \pm 49$ & $6 \pm 4$ \\
Prednisolone & $2(0.5)$ & $112 \pm 68$ & $4 \pm 4$ \\
\hline
\end{tabular}

Values are presented as number (\%) or mean \pm SD. Doses of corticosteroids were converted equivalent to 1 mg of methylprednisolone. 